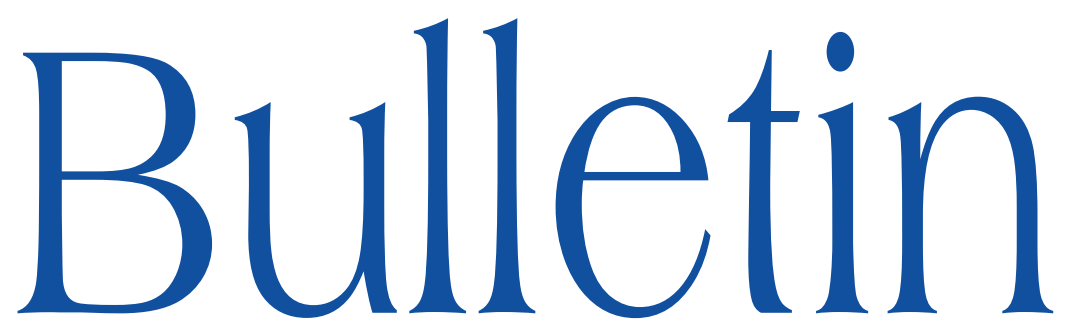

de la SOCIÉTÉ MATHÉMATIQUE DE FRANCE

\title{
ON THE KÄHLER RANK OF COMPACT COMPLEX SURFACES
}

\author{
Matei Toma
}

\section{Tome 136}

Fascicule 2

2008 


\title{
ON THE KÄHLER RANK OF COMPACT COMPLEX SURFACES
}

\author{
BY MATEI TOMA
}

\begin{abstract}
Harvey and Lawson introduced the Kähler rank and computed it in connection to the cone of positive exact currents of bidimension $(1,1)$ for many classes of compact complex surfaces. In this paper we extend these computations to the only further known class of surfaces not considered by them, that of Kato surfaces. Our main tool is the reduction to the dynamics of associated holomorphic contractions $\left(\mathbb{C}^{2}, 0\right) \rightarrow\left(\mathbb{C}^{2}, 0\right)$.

RÉSUmÉ (Sur le rang de Kähler des surfaces complexes compactes)

Harvey et Lawson ont introduit et calculé le rang de Kähler en relation avec le cône des courants positifs fermés de bidimension $(1,1)$ pour beaucoup de classes de surfaces complexes compactes. Dans ce travail nous étendons ces calculs à la seule classe de surfaces connues et qui n'avait pas été considérée par eux, celle des surfaces de Kato. Notre outil principal est la réduction à la dynamique des contractions holomorphes $\left(\mathbb{C}^{2}, 0\right) \rightarrow\left(\mathbb{C}^{2}, 0\right)$ associées.
\end{abstract}

Texte reçu le 23 avril 2007, révisé le 12 juin 2007

Matei Toma, Institut de Mathématiques Élie Cartan, Nancy-Université, B.P. 239, 54506 Vandœuvre-lès-Nancy Cedex, France and Institute of Mathematics of the Romanian Academy • E-mail : toma@iecn.u-nancy.fr • Url : http://www.iecn.unancy.fr/ toma/

2000 Mathematics Subject Classification. - 32J15, 32H50.

Key words and phrases. - Compact complex surface, global spherical shell, closed positive current, iteration of polynomial maps.

The author wishes to thank the Max-Planck-Institut für Mathematik in Bonn and the University of Osnabrück for their hospitality and for financial support during the preparation of this paper. Furthemore he thanks Charles Favre and Karl Oeljeklaus for useful discussions and the referee for his remarks which improved the presentation of the paper. 


\section{Introduction}

In [8] Harvey and Lawson give a characterisation of Kählerianity for compact complex surfaces in terms of existence (or rather non-existence) of closed positive currents which are $(1,1)$-components of a boundary. The authors also investigate and describe the cones formed by such currents for many types of non-Kähler surfaces: elliptic, Hopf, Inoue. Later Lamari proved that every non-Kähler surface admits non-trivial positive $d$-exact currents of bidimension $(1,1)$; cf. [10]. In order to estimate the degree of non-Kählerianity of a compact complex surface smooth positive $d$-exact currents are considered in $[8]$ and the Kähler rank is defined as follows: the Kähler rank is two if the surface admits some Kähler metric, one if it admits some positive $d$-exact $(1,1)$-form with some supplementary property and zero in the remaining case; see the more precise Definition 2. Unfortunately it is not clear whether the Kähler rank is a bimeromorphic invariant.

We consider in this paper instead a bimeromorphic invariant which we call the modified Kähler rank and which we define to be two if the surface is Kähler, one if the cone of positive $d$-exact currents of bidimension $(1,1)$ is larger than a half-line and zero if this cone is a half-line. The two notions agree in the cases considered in [8]. Our main result is the computation of the cones of positive exact $(1,1)$-currents for Kato surfaces. These are surfaces whose minimal models have positive second Betti number and admit a global spherical shell (see Definition 9) and are the only "known" compact complex surfaces not considered in [8]. (We refer the reader to [1] for the general theory of compact complex surfaces.) It turns out that the modified Kähler rank does not coincide with the Kähler rank in general. In order to perform our computations we reduce ourselves to the investigation of plurisubharmonic functions with a certain invariance property with respect to polynomial automorphisms of $\mathbb{C}^{2}$ associated to Kato surfaces. As a corollary we obtain

THEOREM 1. - (a) Every positive d-exact $(1,1)$-current on a Kato surface, and more generally on any "known" non-Kählerian compact complex surface, is a foliated current for some holomorphic foliation of the surface.

(b) All positive d-closed $(1,1)$-currents on the "known" non-Kählerian compact complex surfaces excepting on parabolic Inoue surfaces are foliated currents for some holomorphic foliations.

See Section 3 for definitions.

TOME $136-2008-\mathrm{N}^{\mathrm{O}} 2$ 


\section{The Kähler rank}

We let $X$ be a compact complex surface and denote by $P_{b d y}=P_{b d y}(X)$, $P_{b d y}^{\infty}=P_{b d y}^{\infty}(X)$ the cones of positive currents of bidimension $(1,1)$ which are boundaries (i.e. are $d$-exact), respectively smooth such currents. These objects have been first studied in [8]. In [10] it was shown that $X$ is Kähler if and only if $P_{b d y}(X)$ is trivial. It is easy to see that on a non-Kähler surface every positive $d$-closed differential form of type $(1,1)$ is $d$-exact, cf. [10]. The following definition of Harvey and Lawson gives a measure of non-Kählerianity by looking at the positive closed differential(1,1)-forms on $X$. It is roughly speaking the largest generic rank of a positive closed $(1,1)$-form on $X$.

Definition 2. Let $B(X)=\left\{x \in X \mid \exists \phi \in P_{b d y}^{\infty}(X) \phi_{x} \neq 0\right\}$. The Kähler rank of $X$ is defined to be two if $X$ admits a Kähler metric. If $X$ is non-Kähler the Kähler rank is set to be one when $B(X)$ contains a non-trivial Zariski open subset of $X$ and zero otherwise.

It is not known whether the Kähler rank is a bimeromorphic invariant.

We propose also the following:

Definition 3. - The modified Kähler rank of $X$ is defined to be two when $X$ admits no non-trivial positive exact current of bidimension $(1,1)$, zero when it admits exactly one such current up to a multiplicative constant and one otherwise.

One can show easily that the modified Kähler rank is a bimeromorphic invariant by taking push-down and pull-back of currents through blowing up maps. See the proof of Proposition 7 for a more precise description.

For elliptic surfaces, primary Hopf surfaces and Inoue surfaces one sees that the Kähler rank and the modified Kähler rank coincide using the precise description of $P_{b d y}$ given in [8] in these cases.

Proposition 4. - Let $T$ be a positive exact current of bidimension $(1,1)$ on the compact complex surface $X$. Then there is a representation $\rho: \pi_{1}(X) \rightarrow$ $(\mathbb{R},+)$ and a plurisubharmonic function $u$ on the universal cover $\tilde{X}$ of $X$ such that $T=d d^{c} u$ and $u \circ g=u+\rho(g)$ for all $g \in \pi_{1}(X)$.

The function u can be chosen to be smooth if $T$ is smooth.

Proof. - One has $b_{1}=\operatorname{dim}_{\mathbb{C}} H^{1}(X, \mathbb{C})=\operatorname{dim}_{\mathbb{C}} H^{1}\left(X, \mathcal{O}_{X}\right)+\operatorname{dim}_{\mathbb{C}} H^{0}\left(X, \Omega_{X}^{1}\right)=$ $h^{0,1}+h^{1,0}, \operatorname{cf}[1]$. Denoting the sheaf of closed differential $(1,0)$-forms by $d \mathcal{O}_{X}$ and looking at the long exact cohomology sequence of

$$
0 \rightarrow \mathbb{C}_{X} \rightarrow \mathcal{O}_{X} \rightarrow d \mathcal{O}_{X} \rightarrow 0
$$


one gets an exact sequence

$$
0 \rightarrow H^{0}\left(d \mathcal{O}_{X}\right) \rightarrow H^{1}\left(\mathbb{C}_{X}\right) \rightarrow H^{1}\left(\mathcal{O}_{X}\right) .
$$

By the above equality follows now the surjectivity of the natural map $H^{1}\left(\mathbb{C}_{X}\right) \rightarrow H^{1}\left(\mathcal{O}_{X}\right)$. This is given by mapping a de Rham cohomology class $[\beta]$ of a differential form $\beta=\beta^{1,0}+\beta^{0,1}$ onto the Dolbeault cohomology class of $\beta^{0,1}$.

Let now $T$ be a positive exact current of bidimension $(1,1)$ on $X$. Then $T=d S$ with $S=S^{1,0}+S^{0,1}, S^{1,0}, S^{0,1}$ currents of order zero and bidegree $(1,0)$ and $(0,1)$ respectively, and $S^{1,0}=\bar{S}^{0,1}$. Since $\bar{\partial} S^{0,1}=0, S^{0,1}$ represents a cohomology class in $H^{1}\left(\mathcal{O}_{X}\right)$ and let $\beta=\beta^{1,0}+\beta^{0,1}$ be a closed differential form with $\left[\beta^{0,1}\right]=\left[S^{0,1}\right]$ in $H^{1}\left(\mathcal{O}_{X}\right)$ and $U$ a current of degree 0 on $X$ with $S^{0,1}=\beta^{0,1}+\bar{\partial} U$. The lift $\tilde{\beta}$ of $\beta$ to the universal cover $\tilde{X}$ is $d$-exact and let $f$ be a smooth function on $\tilde{X}$ with $d f=\beta$. In particular $\bar{\partial} f=\beta^{0,1}$. This implies $S^{0,1}=\bar{\partial}(f+U)$ and $T=d S=d(\bar{\partial}(f+U)+\partial(\bar{f}+\bar{U}))=i \partial \bar{\partial}(2 \mathfrak{I m}(f+U))$.

Moreover for $g \in \pi_{1}(X)$ we have $d(f \circ g-f)=0$ hence $f \circ g-f$ must be constant. Set $\rho(g)=2 \mathfrak{I m}(f \circ g-f)$. The current $2 \mathfrak{I m}(f+U)$ is associated to a plurisubharmonic function $u$ on $\tilde{X}$. Since $u-2 \mathfrak{I m} f=2 \mathfrak{I m} U$ comes from $X$ we see that $u$ has the desired automorphy behaviour with respect to the action of $\pi_{1}(X)$.

It is clear that $u$ can be chosen to be smooth when $T$ is smooth.

Definition 5. - We say that an effective reduced divisor $C=C_{1}+\cdots+C_{n}$ on $X$ is a cycle of rational curves if $n \geq 1, C_{1}, \ldots, C_{n}$ are rational curves and either $n=1$ and $C_{1}$ has a node or $n>1$, all components $C_{1}, \ldots, C_{n}$ are smooth and the dual graph of $C$ is cyclic.

Corollary 6. - For a compact complex surface $X$ with a cycle of rational curves $C$ and $b_{1}(X)=1$ the Kähler rank is zero.

Proof. - Under the above hypotheses the natural map $\mathbb{Z} \cong \pi_{1}(C) \rightarrow \pi_{1}(X)$ is an isomorphism by a Theorem of Nakamura, [13]. Let $g$ be a generator of $\pi_{1}(X)$. If the Kähler rank of $X$ were one, we would get a smooth non-constant plurisubharmonic function $u$ on the universal cover $\tilde{X}$ satisfying

$$
u \circ g=u+c
$$

for some constant $c \in \mathbb{R}$ by Proposition 4 . The inverse image $\tilde{C}$ of the cycle of rational curves $C$ of $X$ is an infinite chain of rational curves on $\tilde{X}$. Since $u$ is smooth and constant on each link of this chain we would get $c=0$ hence $u$ would descend to $X$. But here $u$ must be constant contradicting our assumptions.

Proposition 7. - The modified Kähler rank is a bimeromorphic invariant. 
Proof. - It is known that for smooth surfaces the property of being Kähler is invariant under bimeromorphic transformations.

Let now $X$ be non-Kähler and $X^{\prime}$ the blown-up surface at a point $x \in X$. Any plurisubharmonic function $u$ on the universal cover $\tilde{X}$ may be lifted to $\tilde{X}^{\prime}$. Conversely let $u^{\prime}$ be a plurisubharmonic function on $\tilde{X}^{\prime}$. It will be constant on the exceptional divisors of $\tilde{X}^{\prime}$ coming from $X^{\prime} \rightarrow X$. Thus $u^{\prime}$ is the pullback of a plurisubharmonic function on $\tilde{X}$, which satisfies the same invariance condition as $u$ with respect to the action of $\pi_{1}(X) \cong \pi_{1}\left(X^{\prime}\right)$. This gives a bijective correspondence between $P_{b d y}(X)$ and $P_{b d y}\left(X^{\prime}\right)$, hence the invariance of the modified Kähler rank.

From the above argument it also follows that a counterexample to the bimeromorphic invariance of the Kähler rank could only be given by some non-elliptic non-Kähler minimal surface $X$ of Kähler rank zero admitting a continuous plurisubharmonic function $u$ on the universal cover $\tilde{X}$ which is smooth outside a discrete subset of $\tilde{X}$ and exhibits the automorphy behaviour from Proposition 4. From [8] we gather that this is not the case of Hopf surfaces or Inoue surfaces and as we shall see it is not going to be the case of Kato surfaces either.

Finally let us mention the following description of the cone $P(X)$ of positive $d$-closed $(1,1)$-currents:

REMARK 8. - Let $C_{1}, \ldots, C_{n}$ be the irreducible curves of negative selfintersection a non-Kählerian surface $X$ and $\left[C_{1}\right], \ldots,\left[C_{n}\right]$ the corresponding currents of integration. Then

$$
P(X)=P_{b d y}(X)+\sum_{i} \mathbb{R}_{\geq 0}\left[C_{i}\right]
$$

Proof. - Let us denote by $F_{j}$ the irreducible curves of $X$ of zero selfintersection. Since $X$ is non-algebraic it will admit no curve of positive self-intersection. Thus the Siu decomposition of a positive closed current $T$ takes the form:

$$
T=\sum_{i} a_{i}\left[C_{i}\right]+\sum_{j} b_{j}\left[F_{j}\right]+R,
$$

where $a_{i}, b_{j} \in \mathbb{R}$ and $R$ is a positive current whose Lelong level sets are finite. By [11] Prop. 4.3 and its proof $R$ is nef. Since $X$ is non-Kählerian it follows that $R$ must be exact, cf. [11] Thm. 7.1. The integration currents $\left[F_{j}\right]$ are also exact, since $F_{j}^{2}=0$ and the intersection form on $H_{\mathbb{R}}^{1,1}(X)$ is negative definite for a non-Kählerian surface. 


\section{Kato surfaces}

We recall here some facts on Kato surfaces which will be needed in the sequel. We refer the reader to [9], [3], [4], [5], [6] for more details.

Definition 9. - A global spherical shell on a compact complex surface $X$ is the image $\Sigma$ of the sphere $S^{3}$ by a holomorphic embedding of a neighbourhood of $S^{3}$ from $\mathbb{C}^{2}$ into $X$ such that $X \backslash \Sigma$ is connected. We call $X$ a Kato surface if $X$ admits a global spherical shell and the second Betti number of a minimal model of $X$ is positive.

The other minimal surfaces admitting a global spherical shell are the Hopf surfaces. Their second Betti number is zero.

The notion of global spherical shell and a construction method for surfaces with global spherical shells were introduced by Ma. Kato in [9]. An important analytic object associated to this construction method was introduced and studied by Dloussky in [3]. It is the germ of a holomorphic map $\left(\mathbb{C}^{2}, 0\right) \rightarrow$ $\left(\mathbb{C}^{2}, 0\right)$. We shortly recall the facts now.

Take the unit ball $B$ in $\mathbb{C}^{2}$ around the origin and blow-up the origin. Choose a point $P_{1}$ on the exceptional curve $C_{1}$ thus obtained and blow it up again. Continue by blowing up a point on the last created exceptional curve. After $n$ blow-ups one considers the blowing down map $\pi: B^{\prime} \rightarrow B$. The exceptional divisor on $B^{\prime}$ is a tree of $n$ smooth rational curves. The only $(-1)$-curve among them is the last created curve $C_{n}$. Choose a point $P_{n}$ on $C_{n}$, a biholomorphic map $\sigma: \bar{B} \rightarrow \sigma(\bar{B})$ onto a small compact neighbourhood of $P_{n}$ in $B^{\prime}$ and glue the two components of the boundary of $B^{\prime} \backslash \sigma(B)$ by means of $\sigma \circ \pi$. In this way one obtains a minimal compact complex surface $X$. One can show that the image of $S^{3}$ through $\sigma$ is a global spherical shell on $X$ and that $b_{2}(X)=n$. Thus $X$ is a minimal Kato surface.

The images of the exceptional curves are the only (compact) rational curves on $X$. They form an effective reduced divisor which we denote by $D$. Depending on the structure of $D$ one subdivides the class of minimal Kato surfaces into:

1. Enoki surfaces, when $D$ is a cycle of rational curves and $D$ is homologically trivial,

2. intermediate surfaces, when $D$ consists of a cycle of rational curves and of at least one further rational curve attached to the cycle,

3. Inoue-Hirzebruch surfaces, when $D$ consists of one or two cycles of rational curves and $D$ is not homologically trivial.

In particular Kato surfaces admit cycles of rational curves and therefore their Kähler rank is zero.

In the case of Enoki surfaces a further curve might appear. In such a case the curve will be elliptic and the surface is called a parabolic Inoue surface.

TOME $136-2008-\mathrm{N}^{\mathrm{O}} 2$ 
By definition the Dloussky germ associated to $X$ is the germ of the map $f:=\pi \circ \sigma: B \rightarrow B$ around the origin. It can be shown that the conjugacy class of this germ determines the isomorphy class of $X$. We shall denote by $X(f)$ a Kato surface associated to such a germ of holomorphic map $f:\left(\mathbb{C}^{2}, 0\right) \rightarrow$ $\left(\mathbb{C}^{2}, 0\right)$. One can relate certain analytic objects on $X$ to germs of objects on $\left(\mathbb{C}^{2}, 0\right)$ which are invariant under $f$ as follows.

One recovers first the universal cover $\tilde{X}$ of $X$ from the above construction by considering an infinite number of copies $\left(A_{i}\right)_{i \in \mathbb{Z}}$ of $\bar{B}^{\prime} \backslash \sigma(B)$ and by gluing for all $i \in \mathbb{Z}$ the pseudoconvex component of the border $\partial A_{i-1}$ of $A_{i-1}$ to the pseudoconcave component of $\partial A_{i}$ by means of $\sigma \circ \pi$ again. If one glues in this way only the copies $\left(A_{i}\right)_{i \leq 0}$ and then caps the pseudoconcave end of $A_{0}$ by a copy of $B$ using $\sigma$, one obtains a non-compact complex surface $\hat{X}$, a holomorphic map $p: \tilde{X} \rightarrow \hat{X}$, a $(-1)$-curve $\hat{C}$ on $\hat{X}$ and a point $\hat{O}$ on $\hat{C}$ such that $p$ extends the identity map of $\cup_{i \leq 0} A_{i}, \hat{C}$ is the isomorphic image of a rational curve $C$ of $\tilde{X}$ through $p$ and $p$ restricts to an isomorphism $\tilde{X} \backslash p^{-1}(\hat{O}) \rightarrow$ $\hat{X} \backslash\{\hat{O}\}$. In fact $p^{-1}(\hat{O})$ is the union of the infinitely many rational curves appearing after $C$ on $\tilde{X}$ in the "order of creation", cf. [3], Prop. 3.4. Thus $p: \tilde{X} \rightarrow \hat{X}$ can be seen as a blowing down of the infinitely many exceptional curves in $p^{-1}(\hat{O})$. The generator $g$ of $\pi_{1}(X)$ mapping $A_{i}$ to $A_{i+1}$ induces a holomorphic map $\hat{g}: \hat{X} \rightarrow \hat{X}$ with $p \circ g=\hat{g} \circ p$. One sees that $\hat{O}$ is fixed by $\hat{g}$, that $\hat{g}(\hat{C})=\hat{O}$, and that the germ of $\hat{g}$ at $\hat{O}$ is the same as the germ of $\pi \circ \sigma: B \rightarrow B$ at the origin.

Let now $u$ be any plurisubharmonic function on $\tilde{X}$. The restriction of $u$ to $\hat{X} \backslash\{\hat{O}\}$ extends to a plurisubharmonic function $\hat{u}$ on $\hat{X}$, [7]. It is clear that $\hat{u} \circ \hat{g}=\hat{u}-c$ in case $u \circ g=u-c$ for some $c \in \mathbb{R}$. Conversely one gets a plurisubharmonic function $u=\hat{u} \circ p$ starting from $\hat{u}$. In fact, since the germ of $\hat{g}$ around $\hat{O}$ is contracting, it is enough to have only a germ of $\hat{u}$ around $\hat{O}$ satisfying $\hat{u} \circ \hat{g}=\hat{u}-c$ in order to recover $u$ on $\tilde{X}$ with the property $u \circ g=u-c$.

According to [3], [4], [5], [6] we get the following three normal forms for representatives of conjugacy classes of Dloussky germs:

1. in the case of Enoki surfaces

$$
f(z, w)=\left(\alpha z, w z^{s}+Q(z)\right)
$$

where $\alpha \in \Delta^{*}=\Delta \backslash\{0\}, s \geq 1$ and $Q$ is a complex polynomial of degree at most $s$ and with $Q(0)=0$; we have denoted by $\Delta$ the unit disc in $\mathbb{C}$;

2 . in the case of intermediate surfaces

$$
f(z, w)=\left(z^{p}, \lambda w z^{s}+Q(z)\right),
$$

where $p \geq 2, s \geq 1, \lambda \in \mathbb{C}^{*}$ and $Q(z)=\sum_{m=1}^{s} a_{m} z^{m}+a z^{\frac{p s}{p-1}}$ is a complex polynomial with $\operatorname{gcd}\left\{p, m \mid a_{m} \neq 0\right\}=1$ and $a=0$ if $(p-1) \nmid s$ or $\lambda \neq 1$; 
3. in the case of Inoue-Hirzebruch surfaces

$$
f(z, w)=\left(z^{a} w^{b}, z^{c} w^{d}\right)
$$

where the matrix $\left(\begin{array}{ll}a & b \\ c & d\end{array}\right)$ is a product of $b_{2}(X)$ matrices of the form $\left(\begin{array}{ll}0 & 1 \\ 1 & 1\end{array}\right)$ or $\left(\begin{array}{ll}1 & 1 \\ 0 & 1\end{array}\right)$ with at least one factor of the first kind.

In the rest of this paper we shall determine the germs of plurisubharmonic functions around the origin of $\mathbb{C}^{2}$ satisfying $u \circ f=u-c$ for some fixed $c \in \mathbb{R}_{>0}$ and each type of germ $f$ as above.

\section{The main results}

TheOREM 10. - (a) Let $f: \mathbb{C}^{2} \rightarrow \mathbb{C}^{2}$,

$$
f(z, w)=\left(\alpha z, w z^{s}+Q(z)\right)
$$

with $\alpha \in \Delta^{*}, s \geq 1$ and $Q$ is a complex polynomial of degree at most $s$ and with $Q(0)=0$. Let $u: \mathbb{C}^{2} \rightarrow[-\infty, \infty[, u(z, w)=\log |z|$. Then up to some additive constant, $u$ is the only plurisubharmonic function on $\mathbb{C}^{2}$ which satisfies $u \circ f=u+\log |\alpha|$.

(b) On an Enoki surface the integration current on the cycle of rational curves is the only positive exact current of bidimension $(1,1)$ up to multiplicative constants.

Proof. - We start by proving part (b) of the theorem.

Let $C=C_{1}+\cdots+C_{n}$ be the cycle of rational curves on the Enoki surface $X$. Since $C$ is homologically trivial the current of integration $[C]$ along $C$ is a positive $d$-exact current of bidimension $(1,1)$. We denote by $E$ the elliptic curve on $X$ in case it exists.

Let $T$ be an arbitrary positive exact current of bidimension $(1,1)$ on $X$ and $m_{C_{i}}:=\inf \left\{\nu(T, x) \mid x \in C_{i}\right\}, m_{C}=\min m_{C_{i}}, m_{E}$ its generic Lelong numbers along $C_{i}, C$ and $E$ respectively. We denote by $\chi_{A}$ the characteristic function of a subset $A$ of $X$.

The Siu decomposition of $T$ has the form

$$
\begin{gathered}
T=\chi_{E} T+\chi_{C} T+\chi_{X \backslash(C \cup E)} T=m_{E}[E]+\sum_{i} m_{C_{i}}\left[C_{i}\right]+\chi_{X \backslash(C \cup E)} T= \\
=m_{E}[E]+\sum_{i}\left(m_{C_{i}}-m_{C}\right)\left[C_{i}\right]+m_{C}[C]+\chi_{X \backslash(C \cup E)} T
\end{gathered}
$$

(see [2] 6.18, 3.2.4.) Since $C$ is the only homologically trivial effective reduced non-trivial divisor $X$ we get as in Remark 8 that $\chi_{X \backslash(C \cup E)} T$ is exact and that

$$
T=m_{C}[C]+\chi_{X \backslash(C \cup E)} T .
$$

TOME $136-2008-\mathrm{N}^{\mathrm{O}} 2$ 
Hence we may replace $T$ by $\chi_{X \backslash(C \cup E)} T$ which is positive, $d$-exact, has vanishing generic Lelong number along $C$ and is the trivial extension to $X$ of the restriction of $T$ to $X \backslash(C \cup E)$. After normalisation we obtain a corresponding plurisubharmonic function $v$ on $\mathbb{C}^{2}$ satisfying $v \circ f=v+\log |\alpha|$. We denote the current $d d^{c} v$ on $\mathbb{C}^{2}$ by $T$ again. Consequently we have $f^{*} T=T$. Moreover, since $C$ corresponds to the axis $A=\{z=0\} \subset \mathbb{C}^{2}$, the generic Lelong number $m_{A}$ of $T$ along $A$ must vanish. Since $f(A)=\{0\}$ and in general $\nu\left(f^{*} T, x\right) \geq \nu(T, f(x))$ (cf. [12]) it follows that $\nu(T, 0)=0$.

The differential form $d z$ defines a holomorphic foliation on $\mathbb{C}^{2}$ which is invariant by $f$, since $f^{*}(d z)=\alpha d z$.

ClaIM. - $T$ is a foliation current for this foliation.

This means that for any test function $\phi \in \mathcal{C}_{c}^{\infty}\left(\mathbb{C}^{2}\right)$ one has $T(\phi d z \wedge d \bar{z})=0$. As before $T$ is the trivial extension to $\mathbb{C}^{2}$ of its restriction to $\mathbb{C}^{2} \backslash A$. It is therefore enough to check that $T(\phi d z \wedge d \bar{z})=0$ for test functions $\phi$ with support in $\mathbb{C}^{2} \backslash A$.

We have

$$
\begin{gathered}
|T(\phi d z \wedge d \bar{z})|=\left|f^{*} T(\phi d z \wedge d \bar{z})\right|=\left|T\left(\left(f^{-1}\right)^{*}(\phi d z \wedge d \bar{z})\right)\right|= \\
=\left|T\left(\frac{\phi \circ f^{-1}}{|\alpha|^{2}} d z \wedge d \bar{z}\right)\right| \leq \frac{\max |\phi|}{|\alpha|^{2}} 2 \sigma_{T}(f(\operatorname{Supp} \phi))
\end{gathered}
$$

where $\sigma_{T}=T \wedge \frac{i}{2}(d z \wedge d \bar{z}+d w \wedge d \bar{w})$ denotes the trace measure of $T$. Iterating we obtain

$$
|T(\phi d z \wedge d \bar{z})| \leq \frac{\max |\phi|}{|\alpha|^{2 n}} 2 \sigma_{T}\left(f^{n}(\operatorname{Supp} \phi)\right)
$$

for any $n \in \mathbb{N}$.

We now need to estimate how large $f^{n}(B(0, R))$ is. Take $C_{1}=\max \left\{\frac{|Q(z)|}{|z|} \mid z \in\right.$ $\bar{\Delta}$ \}. We denote by $P\left(R_{1}, R_{2}\right)$ the bidisc of radii $R_{1}, R_{2}$ centered at the origin of $\mathbb{C}^{2}$. Then

$$
\begin{aligned}
& f\left(P\left(1, R_{2}\right) \subset P\left(|\alpha|, R_{2}+C_{1}\right),\right. \\
& f^{2}\left(P\left(1, R_{2}\right)\right. \subset P\left(|\alpha|^{2},|\alpha|\left(R_{2}+C_{1}\right)+|\alpha| C_{1}\right) \subset \\
& \subset P\left(|\alpha|^{2},|\alpha|\left(R_{2}+C_{1}\right)+\left(\frac{1}{1-|\alpha|}-1\right) C_{1}\right),
\end{aligned}
$$




$$
\begin{aligned}
& f^{3}\left(P\left(1, R_{2}\right)\right. \subset P\left(|\alpha|^{3},|\alpha|^{3}\left(R_{2}+C_{1}\right)+\frac{|\alpha|^{2}}{1-|\alpha|} \mid C_{1}\right) \subset \\
& \subset P\left(|\alpha|^{3},|\alpha|^{3}\left(R_{2}+C_{1}\right)+\left(\frac{1}{1-|\alpha|}-1\right) C_{1}\right), \\
& f^{4}\left(P\left(1, R_{2}\right)\right. \subset P\left(|\alpha|^{4},|\alpha|^{6}\left(R_{2}+C_{1}\right)+\frac{|\alpha|^{3}}{1-|\alpha|} \mid C_{1}\right) \subset \\
& \subset P\left(|\alpha|^{4},|\alpha|^{6}\left(R_{2}+C_{1}\right)+\left(\frac{1}{1-|\alpha|}-1\right) C_{1}\right),
\end{aligned}
$$

and further

$$
f^{n}\left(P\left(1, R_{2}\right) \subset P\left(|\alpha|^{n},|\alpha|^{\frac{n(n-1)}{2}}\left(R_{2}+C_{1}\right)+\frac{|\alpha|^{n-1}}{1-|\alpha|} \mid C_{1}\right) .\right.
$$

From this and from (1) it follows that there is a constant $C_{2}$ such that

$$
|T(\phi d z \wedge d \bar{z})| \leq C_{2} \max |\phi| \frac{\sigma_{T}\left(B\left(0,|\alpha|^{n}\right)\right.}{|\alpha|^{2 n}},
$$

for all $n$ sufficiently large. But

$$
\lim _{n \rightarrow \infty} \frac{\sigma_{T}\left(B\left(0,|\alpha|^{n}\right)\right.}{|\alpha|^{2 n}}=\pi \nu(T, 0)=0
$$

and the claim follows.

Using the claim it can be easily shown that $T$ is invariant under translations in the $w$-direction, hence $T=p r_{1}^{*} S$, where $S$ is a positive current on $\mathbb{C}$ of dimension zero and $p r_{1}: \mathbb{C}^{2} \rightarrow \mathbb{C}$ denotes the first projection. Set $f_{1}: \mathbb{C} \rightarrow \mathbb{C}$, $f_{1}(z)=\alpha z$. Then $p r_{1} \circ f=f_{1} \circ p r_{1}$, hence $f_{1}^{*}(S)=S$. The current $S$ is of the form $\mu i d z \wedge d \bar{z}$, where $\mu$ is a positive measure on $\mathbb{C}$. Denote by $\Delta(r)$ the disc of radius $r$ around the origin of $\mathbb{C}$. The invariance property of $S$ implies

$$
\mu(\Delta(r))=\mu(\Delta(|\alpha| r))=\mu\left(\Delta\left(|\alpha|^{n} r\right)\right)
$$

for all $n \in \mathbb{N}$ and $r \in \mathbb{R}_{>0}$. This entails that $S$ is supported at the origin of $\mathbb{C}$ and hence that $T=0$, since $T$ is not carried by $A$.

We now turn to part (a) of the theorem. Let $v$ be a plurisubharmonic function on $\mathbb{C}^{2}$ satisfying $v \circ f=f+\log |\alpha|$. By part (b) of the theorem we see that $d d^{c} v \leq d d^{c} u$ or $d d^{c} u \leq d d^{c} v$. But then $u-v$ or $v-u$ would give a plurisubharmonic function on $X$ which has to be constant.

Notation. - For $\alpha \in \mathbb{R}_{>0}$ we denote by $K_{\alpha}$ the set of continuous $\alpha$-periodic functions $\psi: \mathbb{R} \rightarrow \mathbb{R}$ which fulfil the inequality

$$
-\psi^{\prime \prime}+\psi^{\prime}+1 \geq 0
$$

in generalised sense. 
Notice that $K_{\alpha}$ is infinite dimensional: for any $\alpha$-periodic smooth function $\phi: \mathbb{R} \rightarrow \mathbb{R}$ and for any small enough factor $\varepsilon \in \mathbb{R}_{>0}$ one has $\varepsilon \phi \in K_{\alpha}$.

TheOREM 11. - (a) Let $f: \Delta \times \mathbb{C} \rightarrow \Delta \times \mathbb{C}$,

$$
f(z, w)=\left(z^{p}, \lambda w z^{s}+Q(z)\right)
$$

where $p \geq 2, s \geq 1, \lambda \in \mathbb{C}^{*}$ and $Q(z)=\sum_{m=1}^{s} a_{m} z^{m}+a z^{\frac{p s}{p-1}}$ is a complex polynomial with $\operatorname{gcd}\left\{p, m \mid a_{m} \neq 0\right\}=1$ and $a=0$ if $(p-1) \nmid s$ or $\lambda \neq 1$. The plurisubharmonic functions $u: \Delta \times \mathbb{C} \rightarrow[-\infty, \infty[$ which satisfy $u \circ f=u-\log p$ are precisely the functions of the form

$$
u(z, w)=-\log (-\log |z|)-\psi(\log (-\log |z|))
$$

for $\psi \in K_{\log p}$.

(b) The cone of positive exact currents of bidimension $(1,1)$ on the intermediate surface $X(f)$ corresponds bijectively to the cone of currents of the form $c d d^{c} u$ on $\Delta \times \mathbb{C}$ for $u$ as above and $c \in \mathbb{R}_{>0}$. In particular, the modified Kähler rank of $X(f)$ is one.

Proof. - There are no homologically trivial divisors on $X(f)$ this time (excepting 0 of course). As in the case of Enoki surfaces we reduce ourselves to the investigation of closed positive currents $T$ on $\Delta \times \mathbb{C}$ with vanishing Lelong number at the origin and satisfying $f^{*} T=T$. Moreover we may again suppose that $T$ is the extension to $\Delta \times \mathbb{C}$ of its restriction to $\Delta^{*} \times \mathbb{C}$.

The differential form $d z$ defines a holomorphic foliation on $\Delta \times \mathbb{C}$ which is invariant under $f$.

We start again by showing that $T$ is a foliation current for this foliation. For this it is enough to check that the measure

$$
i d z \wedge d \bar{z} \wedge T
$$

vanishes on $\Delta^{*} \times \mathbb{C}$. We use the invariance of $T$ by $f$ again.

Take $0<r_{1}<r_{2}<1, r^{\prime}>0, D:=\left(\Delta\left(r_{2}\right) \backslash \Delta\left(r_{1}\right)\right) \times \Delta\left(r^{\prime}\right), D^{\prime}:=$ $f^{-n}\left(f^{n}(D)\right)$ and $A\left(r_{1}, r_{2}\right)=A\left(r_{1}, r_{2}, r^{\prime}\right):=(i d z \wedge d \bar{z} \wedge T)(D)$ the measure of the set $D$. We have of course $D^{\prime} \supset D$. Since $f: \Delta^{*} \times \mathbb{C} \rightarrow \Delta^{*} \times \mathbb{C}$ is $p$ to 1 we get

$$
\begin{gathered}
(i d z \wedge d \bar{z} \wedge T)\left(f^{n}(D)\right)=p^{n}\left(i|z|^{2\left(p^{n}-1\right)} d z \wedge d \bar{z} \wedge T\right)\left(D^{\prime}\right) \geq \\
\geq p^{n}\left(i|z|^{2 p^{n}} d z \wedge d \bar{z} \wedge T\right)\left(D^{\prime}\right) \geq p^{n} r_{1}^{2 p^{n}} A\left(r_{1}, r_{2}\right)
\end{gathered}
$$

Hence

$$
A\left(r_{1}, r_{2}\right) \leq \frac{1}{p^{n}}\left(\frac{r_{2}}{r_{1}}\right)^{2 p^{n}} \frac{i\left(\partial \bar{\partial}\left(|z|^{2}+|w|^{2 p}\right) \wedge T\right)\left(f^{n}(D)\right)}{\pi r_{2}^{2 p^{n}}}
$$


As before we need to estimate the width of $f^{n}(D)$. Let $C_{1}=\max \left\{\frac{|Q(z)|}{|z|} \mid z \in\right.$ $\bar{\Delta}\}$ and $C_{2}=\max (1,|\lambda|)$. For $0<r<1,0<r^{\prime}$ we see that

$$
\begin{aligned}
f\left(P\left(r, r^{\prime}\right)\right) & \subset P\left(r^{p}, r C_{2}\left(r^{\prime}+\frac{C_{1}}{C_{2}}\right)\right) \subset P\left(r^{p}, r C_{2}\left(r^{\prime}+\frac{C_{1}}{(1-r) C_{2}}\right)\right), \\
f^{2}\left(P\left(r, r^{\prime}\right)\right) & \subset P\left(r^{p^{2}}, r^{p+1} C_{2}^{2} r^{\prime}+\frac{r^{p-1} C_{2}^{2} C_{1}}{(1-r) C_{2}}\right) \\
& \subset P\left(r^{p^{2}}, r^{p-1} C_{2}^{2}\left(r^{\prime}+\frac{C_{1}}{(1-r) C_{2}}\right)\right), \\
f^{3}\left(P\left(r, r^{\prime}\right)\right) & \subset P\left(r^{p^{3}}, r^{p^{2}} C_{2}\left(r^{p-1} C_{2}^{2}\left(r^{\prime}+\frac{C_{1}}{(1-r) C_{2}}\right)+\frac{C_{1}}{C_{2}}\right)\right) \\
& \subset P\left(r^{p^{3}}, r^{p^{2}+p-1} C_{2}^{3} r^{\prime}+\frac{r^{p^{2}-1} C_{2}^{3} C_{1}}{(1-r) C_{2}}\right) .
\end{aligned}
$$

For sufficiently large $n$ we thus obtain

$$
f^{n}\left(P\left(r, r^{\prime}\right)\right) \subset\left\{|z|^{2}+|w|^{2 p}<2 C_{1} r^{p^{n}}\right\}
$$

which in combination with the inequality (2) gives

$$
A\left(r_{1}, r_{2}\right) \leq \frac{1}{p^{n}}\left(\frac{r_{2}}{r_{1}}\right)^{2 p^{n}} \frac{2 i\left(\partial \bar{\partial}\left(|z|^{2}+|w|^{2 p}\right) \wedge T\right)\left(\left\{|z|^{2}+|w|^{2 p}<2 C_{1} r_{2}^{p^{n}}\right\}\right)}{\pi r_{2}^{2 p^{n}}} .
$$

But the factor

$$
\frac{2 i\left(\partial \bar{\partial}\left(|z|^{2}+|w|^{2 p}\right) \wedge T\right)\left(\left\{|z|^{2}+|w|^{2 p}<2 C_{1} r_{2}^{p^{n}}\right\}\right)}{\pi r_{2}^{2 p^{n}}}
$$

converges to $4 C_{1} \nu(T, \phi, 0)$, where $\nu(T, \phi, 0)$ is the Lelong number with respect to $\phi=\log \left(|z|^{2}+|w|^{2 p}\right)$. This Lelong number vanishes by the Comparison Theorem for Lelong numbers [2], since the usual Lelong number vanishes. So for any $\varepsilon>0$ we can find some $N \in \mathbb{N}$ such that

$$
A\left(r_{1}, r_{2}\right) \leq \frac{1}{p^{n}}\left(\frac{r_{2}}{r_{1}}\right)^{2 p^{n}} \varepsilon
$$

for all $n \geq N$. Moreover this inequality holds also for smaller $r_{1}$ and $r_{2}$.

Let

$$
\delta=\left(\frac{r_{2}}{r_{1}}\right)^{\frac{1}{2 p^{n}}}
$$

and look at the division $\left(r_{1}, \delta r_{1}, \delta^{2} r_{1}, \ldots, \delta^{2 p^{n}} r_{1}=r_{2}\right)$ of the interval $\left[r_{1}, r_{2}\right]$. We have seen that $A\left(\delta^{i} r_{1}, \delta^{i+1} r_{1}\right) \leq \frac{1}{p^{n}} \delta^{2 p^{n}} \varepsilon=\frac{1}{p^{n}} \frac{r_{2}}{r_{1}} \varepsilon$ hence $A\left(r_{1}, r_{2}\right)=$ $\sum_{i=1}^{2 p^{n}} A\left(\delta^{i-1} r_{1}, \delta^{i} r_{1}\right) \leq 2 \frac{r_{2}}{r_{1}} \varepsilon$, which proves that $T$ is a foliation current.

As in the proof of Theorem 10, $T$ is invariant under translations in the $w$ direction, hence $T=p r_{1}^{*} S$, where $S$ is a positive current on $\Delta$ of dimension zero and $p r_{1}: \Delta \times \mathbb{C} \rightarrow \Delta$ is the first projection. On the other hand $T=d d^{c} u$, 
where $\mathrm{u}$ is a plurisubharmonic function on $\Delta \times \mathbb{C}$ with $u \circ f=u-\log p$. The restriction of $u$ to a general leaf $\{z=$ const. $\}$ of our foliation is a harmonic function in $w$. We shall show that $\frac{\partial u}{\partial w}=0$, the case of $\frac{\partial u}{\partial \bar{w}}=0$ being similar.

Since $T$ is a foliation current one has that $\frac{\partial^{2} u}{\partial z \partial w}=\frac{\partial^{2} u}{\partial z \partial w}=0$ as distributions. In particular $\frac{\partial u}{\partial w}=0$ is invariant by translations in the $z$-directions. The invariance condition by translations in $w$ implies that

$$
\lambda z^{s} \frac{\partial u}{\partial w}(f(z, w))=\frac{\partial u}{\partial w}(z, w)
$$

Take now a small non-zero solution $z^{\prime}$ of the equation

$$
w=\lambda z^{s} w+Q(z)
$$

for fixed small $w, w \neq 0$. Using the invariance in the $z$-direction together with (3) and (4) we obtain

$$
\frac{\partial u}{\partial w}(z, w)=\frac{\partial u}{\partial w}\left(z^{\prime}, w\right)=\lambda\left(z^{\prime}\right)^{s} \frac{\partial u}{\partial w}\left(\left(z^{\prime}\right)^{p}, w\right)=\lambda\left(z^{\prime}\right)^{s} \frac{\partial u}{\partial w}(z, w),
$$

hence $\frac{\partial u}{\partial w}(z, w)=0$ for small $w$. But for large $w$ the vanishing holds as well since we can iterate on (3).

Thus $u$ is constant on the leaves $\{z=$ const. $\}$ and therefore descends to a subharmonic function $u$ on $\Delta$ fulfilling the condition $u\left(z^{p}\right)=u(z)-\log p$ for all $z \in \Delta$.

Take now $z \in \Delta$ and a $p^{n}$-th root of unity $\theta$. The invariance condition implies $u(z)=u\left(z^{p^{n}}\right)+n \log p=u(\theta z)$ hence each value $u(z)$ is attained on a dense subset of the circle $\{|z|=$ const. $\}$. By semi-continuity it follows that $u$ depends only on $r=|z|$.

Let $v=\left.u\right|_{[0,1[}$. By the maximum principle and the invariance condition on $u$ one sees that $v$ is strictly increasing. Using this, the semi-continuity of $u$ and the definition of subharmonicity (cf. [2] 1.4.13.b) one infers that $v$ is continuous. The Laplace operator takes the form $\frac{\partial^{2}}{\partial r^{2}}+\frac{1}{r} \frac{\partial}{\partial r}+\frac{1}{r^{2}} \frac{\partial^{2}}{\partial \phi^{2}}$ in polar coordinates $(r, \phi)$. We thus reduce ourselves to the search of continuous functions $v:[0,1[\rightarrow[-\infty, \infty[$ which satisfy

$$
v\left(r^{p}\right)=v(r)-\log p
$$

and

$$
v^{\prime \prime}(r)+\frac{1}{r} v^{\prime}(r) \geq 0 .
$$

Let $h:]-\infty, \infty] \rightarrow[0,1[, h(t)=\exp (-\exp t)$ and $\psi=-v \circ h-h$. The conditions (5) and (6) translate into

$$
\begin{gathered}
\psi(t+\log p)=\psi(t), \\
-\psi^{\prime \prime}+\psi^{\prime}+1 \geq 0
\end{gathered}
$$


and thus $\psi \in K_{\log p}$ and the theorem is proved.

For the case of Inoue-Hirzebruch surfaces we need some preparations. Let $f: \mathbb{C}^{2} \rightarrow \mathbb{C}^{2}, f(z, w)=\left(z^{a} w^{b}, z^{c} w^{d}\right)$, and denote by $A$ the matrix $\left(\begin{array}{ll}a & b \\ c & d\end{array}\right)$. We suppose that $A$ is a product of matrices of the form $\left(\begin{array}{ll}0 & 1 \\ 1 & 1\end{array}\right)$ or $\left(\begin{array}{ll}1 & 1 \\ 0 & 1\end{array}\right)$ with at least one factor of the first kind. We have $\operatorname{det}(A)= \pm 1$ and by Lemma 2.2 from [4], $\operatorname{trace}(A)>2$ unless $A=\left(\begin{array}{ll}0 & 1 \\ 1 & 1\end{array}\right)$ or $A=\left(\begin{array}{ll}0 & 1 \\ 1 & 2\end{array}\right)$. In any case one sees that the eigenvalues $\lambda_{1}, \lambda_{2}$ of $A^{t}$ are real, irrational and one of them is larger than 1 . We set $\lambda=\lambda_{1}>1$. Let $(\alpha, \beta)=\left(\alpha_{1}, \beta_{1}\right),\left(\alpha_{2}, \beta_{2}\right)$ be eigenvectors associated to $\lambda_{1}$ and $\lambda_{2}$ respectively. An easy computation shows that $\alpha \beta=\alpha_{1} \beta_{1}>0$, $\alpha_{2} \beta_{2}<0$. We choose $\alpha, \beta, \alpha_{2} \in \mathbb{R}_{>0}$ and set

$$
\begin{gathered}
\phi=\phi_{1}=\alpha \log |z|+\beta \log |w|, \phi_{2}=\alpha_{2} \log |z|+\beta_{2} \log |w|, \\
U:=\{\phi<0\} \subset \mathbb{C}^{2} .
\end{gathered}
$$

Then $\phi_{i} \circ f=\lambda_{i} \phi_{i}$ for $i=1,2$.

Now we can state

Theorem 12. - (a) For $A, \lambda, \alpha, \beta$ as above let $\phi=\alpha \log |z|+\beta \log |w|$, $U=\phi^{-1}([-\infty, 0[), f: U \rightarrow U$,

$$
f(z, w)=\left(z^{a} w^{b}, z^{c} w^{d}\right) .
$$

Then the plurisubharmonic functions $u$ on $U$ which satisfy $u \circ f=u-\log \lambda$ are precisely the functions of the form

$$
u=-\log (-\phi)-\psi(\log (-\phi)),
$$

for $\psi \in K_{\log p}$.

(b) The cone of positive exact currents of bidimension $(1,1)$ on the InoueHirzebruch surface $X(f)$ corresponds bijectively to the cone of currents of the form $c d d^{c} u$ on $U$ for $u$ as above and $c \in \mathbb{R}_{>0}$. In particular, the modified Kähler rank of $X(f)$ is one.

Proof. - As in the proof of Theorem 11 we reduce ourselves to the investigation of currents $T$ on $U$ of the form $T=d d^{c} u$ with $u$ plurisubharmonic on $U$ and such that $u \circ f=u-\log \lambda$ and $\nu(T, 0)=0$. Moreover we may suppose that $T$ is the extension to $U$ of its restriction to $U \cap\left(\mathbb{C}^{*} \times \mathbb{C}^{*}\right)$.

There is again a holomorphic foliation on $U$, this time singular, which will be shown to be compatible with $T$. On $U \cap\left(\mathbb{C}^{*} \times \mathbb{C}^{*}\right)$ this foliation is induced by the smooth positive $(1,1)$-form $d d^{c} v$ where $v:=-\log (-\phi)$. As before it is enough to show that the measure $d d^{c} v \wedge T$ is zero on any compact subset of $U \cap\left(\mathbb{C}^{*} \times \mathbb{C}^{*}\right)$. 
For a better visualisation of the present situation we introduce the map $E$ : $\mathbb{C} \times \mathbb{C} \rightarrow \mathbb{C}^{*} \times \mathbb{C}^{*}, E(\zeta, \omega)=(\exp \zeta, \exp \omega)$. We denote the linear automorphism induced by $A$ on $\mathbb{C}^{2}$ again by $A$ and get the relations

$$
\begin{gathered}
f \circ E=E \circ A, \\
\phi \circ E(\zeta, \omega)=\alpha_{i} \mathfrak{R e} \zeta+\beta_{i} \mathfrak{R e} \omega, i=1,2 .
\end{gathered}
$$

In particular $E^{-1}(U)=\{\alpha \mathfrak{R e} \zeta+\beta \Re \mathfrak{R} \omega<0\}$.

We also see that $U$ is covered by compact subsets of the form $D=$ $D\left(c_{1}, \delta, c_{2}\right):=\phi_{1}^{-1}\left(\left[-c_{1} \delta,-c_{1}\right]\right) \cap \phi_{2}^{-1}\left(\left[-c_{2}, c_{2}\right]\right)$ for $c_{1}, c_{2} \in \mathbb{R}_{>0}, \delta \in[1, \infty[$. Since both $T$ and $d d^{c} v$ are invariant by $f$ we have

$$
\begin{gathered}
A\left(c_{1}, \delta\right)=A\left(c_{1}, \delta, c_{2}\right):=\left(d d^{c} v \wedge T\right)\left(D\left(c_{1}, \delta, c_{2}\right)\right)= \\
\left.=\left(d d^{c} v \wedge T\right) f^{n}\left(D\left(c_{1}, \delta, c_{2}\right)\right)\right)=\left(d d^{c} v \wedge T\right)\left(D\left(\lambda^{n} c_{1}, \delta, \lambda^{-n} c_{2}\right)\right) .
\end{gathered}
$$

We need to estimate $d d^{c} v$ on $f^{n}(D)$. On $D$ we have

$$
\begin{gathered}
-c_{1} \delta \leq \alpha_{1} \log |z|+\beta_{1} \log |w| \leq-c_{1}, \\
-c_{2} \leq \alpha_{2} \log |z|+\beta_{2} \log |w| \leq c_{2},
\end{gathered}
$$

hence

$$
\begin{gathered}
\exp \left(\frac{c_{1} \delta \beta_{2}-c_{2} \beta_{1}}{\alpha_{2} \beta_{1}-\alpha_{1} \beta_{2}}\right) \leq|z| \leq \exp \left(\frac{c_{1} \beta_{2}+c_{2} \beta_{1}}{\alpha_{2} \beta_{1}-\alpha_{1} \beta_{2}}\right), \\
\exp \left(\frac{-c_{1} \delta \alpha_{2}-c_{2} \alpha_{1}}{\alpha_{2} \beta_{1}-\alpha_{1} \beta_{2}}\right) \leq|w| \leq \exp \left(\frac{-c_{1} \alpha_{2}+c_{2} \alpha_{1}}{\alpha_{2} \beta_{1}-\alpha_{1} \beta_{2}}\right) .
\end{gathered}
$$

Therefore we get on $f^{n}(D)$

$$
\begin{gathered}
\exp \left(\frac{\lambda^{n} c_{1} \delta \beta_{2}-\lambda^{-n} c_{2} \beta_{1}}{\alpha_{2} \beta_{1}-\alpha_{1} \beta_{2}}\right) \leq|z| \leq \exp \left(\frac{\lambda^{n} c_{1} \beta_{2}+\lambda^{-n} c_{2} \beta_{1}}{\alpha_{2} \beta_{1}-\alpha_{1} \beta_{2}}\right), \\
\exp \left(\frac{-\lambda^{n} c_{1} \delta \alpha_{2}-\lambda^{-n} c_{2} \alpha_{1}}{\alpha_{2} \beta_{1}-\alpha_{1} \beta_{2}}\right) \leq|w| \leq \exp \left(\frac{-\lambda^{n} c_{1} \alpha_{2}+\lambda^{-n} c_{2} \alpha_{1}}{\alpha_{2} \beta_{1}-\alpha_{1} \beta_{2}}\right),
\end{gathered}
$$

hence $f^{n}(D) \subset B\left(0, r_{n}\right)$, where

$$
r_{n}^{2}=\exp \left(2 \frac{\lambda^{n} c_{1} \beta_{2}+\lambda^{-n} c_{2} \beta_{1}}{\alpha_{2} \beta_{1}-\alpha_{1} \beta_{2}}\right)+\exp \left(2 \frac{-\lambda^{n} c_{1} \alpha_{2}+\lambda^{-n} c_{2} \alpha_{1}}{\alpha_{2} \beta_{1}-\alpha_{1} \beta_{2}}\right) .
$$

Now on $D$ again

$$
\begin{aligned}
& i \partial \bar{\partial} v=\frac{i \partial \phi \wedge \bar{\partial} \phi}{\phi^{2}}=\frac{i\left(\alpha \frac{d z}{z}+\beta \frac{d w}{w}\right) \wedge\left(\alpha \frac{d \bar{z}}{\bar{z}}+\beta \frac{d \bar{w}}{\bar{w}}\right)}{4 \phi^{2}} \leq \\
& \leq \frac{C_{1}}{\min \left\{|z|^{2},|w|^{2} \mid(z, w) \in D\right\}} \frac{i(d z \wedge d \bar{z}+d w \wedge d \bar{w})}{\phi^{2}}
\end{aligned}
$$

where $C_{1}$ is a constant not depending on $D$. This implies

$$
A\left(c_{1}, \delta\right) \leq \frac{C_{1} r_{n}^{2}}{\min \left\{\phi^{2}(z, w)|z|^{2}, \phi^{2}(z, w)|w|^{2} \mid(z, w) \in f^{n}(D)\right\}} \frac{\sigma_{T}\left(B\left(0, r_{n}^{2}\right)\right)}{r_{n}^{2}} .
$$

BULLETIN DE LA SOCIÉtÉ MATHÉMATIQUE DE FRANCE 
As in the previous cases $\frac{\sigma_{T}\left(B\left(0, r_{n}^{2}\right)\right)}{r_{n}^{2}}$ converges to zero, therefore for any $\varepsilon>0$ we get $\frac{C_{1} \sigma_{T}\left(B\left(0, r_{n}^{2}\right)\right)}{r_{n}^{2}}<\varepsilon$ as soon as $n$ is large enough. Setting $C_{2}=\frac{2 c_{1} \max \left\{\alpha_{1},-\beta_{2}\right\}}{\alpha_{2} \beta_{1}-\alpha_{1} \beta_{2}}+1$ we get by our estimates $r_{n}^{2} \leq \exp \left(-\lambda^{n} C_{2}\right)$ and $\min \left\{|z|^{2},|w|^{2} \mid(z, w) \in f^{n}(D)\right\} \geq \exp \left(-\lambda^{n} C_{2} \delta\right)$, hence

$$
A\left(c_{1}, \delta\right) \leq \frac{\exp \left(\lambda^{n} C_{2}(\delta-1)\right)}{\lambda^{2 n}} \frac{\varepsilon}{c_{1}^{2}} .
$$

Set $m=\left\lfloor\lambda^{2 n}\right\rfloor$ and consider the division $\left(1, \exp \frac{1}{m}, \exp \frac{2}{m}, \ldots, \exp \frac{m}{m}=e\right)$ of the interval $[1, e]$. For any subinterval our estimates hold with $e C_{2}$ instead of $C_{2}$, hence

$$
\begin{gathered}
A\left(c_{1}, e, c_{2}\right)=\sum_{i=1}^{m} A\left(c_{1} \exp \frac{i-1}{m}, \exp \frac{1}{m}, c_{2}\right) \leq \\
\leq m \frac{\exp \left(\lambda^{n} C_{2}\left(\exp \frac{1}{m}-1\right)\right)}{\lambda^{2 n}} \frac{\varepsilon}{c_{1}^{2}} \leq \exp \left(C_{2} e \frac{\exp \frac{1}{m}-1}{\frac{1}{m}}\right) \frac{\varepsilon}{c_{1}^{2}}
\end{gathered}
$$

and the last term converges to $\exp \left(C_{2} e\right) \frac{\varepsilon}{c_{1}^{2}}$ showing that $A\left(c_{1}, e, c_{2}\right)=0$.

As in the proof of Theorem 11 we next check that $u$ is constant on the leaves of the foliation given by $\alpha \frac{d z}{z}+\beta \frac{d w}{w}$ on $U \cap\left(\mathbb{C}^{*} \times \mathbb{C}^{*}\right)$.

Indeed, for $V:=u \circ E$ one has $V \circ A=V-\log \lambda$ on $\{\alpha \mathfrak{R e} \zeta+\beta \mathfrak{R e} \omega<0\}$ and $d d^{c} V$ is a foliation current for the foliation given by $\alpha d \zeta+\beta d \omega$.

We consider a linear change of coordinates which diagonalizes $A$ on $\mathbb{C}^{2}$ leading to $A(\xi, \tau)=\left(\lambda \xi, \lambda^{-1} \tau\right)$. Keeping the notation $V$ for $V$ after this coordinate change we notice that $V$ restricted to the leaves $\{\xi=$ const. $\}$ is harmonic and that $\frac{\partial V}{\partial \tau}, \frac{\partial V}{\partial \bar{\tau}}$ are invariant by translations in the $\xi$-direction. As in the case of intermediate surfaces we get

$$
\frac{1}{\lambda} \frac{\partial V}{\partial \tau}\left(\xi, \frac{1}{\lambda} \tau\right)=\frac{1}{\lambda} \frac{\partial V}{\partial \tau}\left(\lambda \xi, \frac{1}{\lambda} \tau\right)=\frac{\partial V}{\partial \tau}(\xi, \tau) .
$$

Iterating this relation and using the continuity of $\frac{\partial V}{\partial \tau}$ on $\{\xi=$ const. $\}$ we obtain

$$
\frac{\partial V}{\partial \tau}\left(\xi, \frac{1}{\lambda^{n}} \tau\right)=\lambda^{n} \frac{\partial V}{\partial \tau}(\xi, \tau)
$$

which forces $\frac{\partial V}{\partial \tau}$ to vanish. Similarly $\frac{\partial V}{\partial \bar{\tau}}=0$. Thus $V$ is constant on the leaves $\{\xi=$ const. $\}$.

Returning now to the coordinates $(\zeta, \omega)$ on $\mathbb{C}^{2}$ we shall show that $V$ only depends on $\mathfrak{R e} \zeta$ and $\mathfrak{R e} \omega$. The function $V$ is doubly periodic in $\mathfrak{I m} \zeta, \mathfrak{I m} \omega$ since $V=u \circ E$. Fix $\mathfrak{R e} \zeta$ and $\mathfrak{R e} \omega$ and look at a leaf $\{\alpha \zeta+\beta \omega=$ const. $\}$. Under this restriction the imaginary parts must satisfy some relation $\alpha \mathfrak{I m} \zeta+\beta \mathfrak{I m} \omega=$ const. which describes a dense subset in the "torus of the imaginary parts" $\mathbb{R}^{2} /(2 \pi \mathbb{Z})^{2}$ since $\alpha / \beta$ is irrational. On this subset $V$ is constant and by semicontinuity it must be constant on the whole torus. 
Switching once more to coordinates $(\xi, \tau)$ we see that $V$ depends on $\mathfrak{R e} \xi$ alone. We have reduced ourselves in this way to the search of continuous functions $v: \mathbb{R}_{<0} \rightarrow \mathbb{R}$ subject to the conditions

$$
\begin{gathered}
v(\lambda t)=v(t)-\log \lambda, \forall t \in \mathbb{R}_{<0}, \\
v^{\prime \prime} \geq 0 .
\end{gathered}
$$

Remark that the continuity of $v$ can be deduced as in Theorem 11 after first restricting $u$ to the line $\{z=w\}$.

Take now $h: \mathbb{R} \rightarrow \mathbb{R}_{<0}, h(s)=-\exp (s)$ and $\psi=-v \circ h-h$. Then the conditions on $v$ translate into $\psi \in K_{\log \lambda}$. Thus $u=-\log (-\phi)-\psi(\log (-\phi))$ and the proof is finished.

Finally, the first part of Theorem 1 is a direct consequence of the description of $P_{b d y}(X)$ given in [8] and in the above Theorems. For the second part it suffices to apply Remark 8 and to notice that the only case of a curve on a "known" non-Kählerian surface, which is not invariant under a holomorphic foliation, is that of the elliptic curve on a parabolic Inoue surface.

\section{BIBLIOGRAPHY}

[1] W. P. Barth, K. Hulek, C. A. M. Peters \& A. Van de Ven Compact complex surfaces, second ed., Ergebnisse der Mathematik und ihrer Grenzgebiete. 3. Folge, vol. 4, Springer, 2004.

[2] J.-P. Demailly - "Complex analytic and algebraic geometry", http:// ww-fourier.ujf-grenoble.fr/ demailly/manuscripts/agbook.pdf, 2007.

[3] G. Dloussky - "Structure des surfaces de Kato", Mém. Soc. Math. France (N.S.) (1984), p. 120.

[4] _ " "Sur la classification des germes d'applications holomorphes contractantes", Math. Ann. 280 (1988), p. 649-661.

[5] G. Dloussky \& K. Oeljeklaus - "Vector fields and foliations on compact surfaces of class $\mathrm{VII}_{0} "$, Ann. Inst. Fourier (Grenoble) 49 (1999), p. 1503-1545.

[6] C. FAVRE - "Classification of 2-dimensional contracting rigid germs and Kato surfaces. I", J. Math. Pures Appl. (9) 79 (2000), p. 475-514.

[7] H. Grauert \& R. Remmert - "Plurisubharmonische Funktionen in komplexen Räumen", Math. Z. 65 (1956), p. 175-194.

[8] R. HARVEy \& H. B. LAWSON, JR. - "An intrinsic characterization of Kähler manifolds", Invent. Math. 74 (1983), p. 169-198. 
[9] M. Kato - "Compact complex manifolds containing "global" spherical shells", Proc. Japan Acad. 53 (1977), p. 15-16.

[10] A. Lamari - "Courants kählériens et surfaces compactes", Ann. Inst. Fourier (Grenoble) 49 (1999), p. 263-285.

[11] _ _ "Le cône kählérien d'une surface", J. Math. Pures Appl. (9) 78 (1999), p. 249-263.

[12] M. MEO - "Image inverse d'un courant positif fermé par une application analytique surjective", C. R. Acad. Sci. Paris Sér. I Math. 322 (1996), p. 1141-1144.

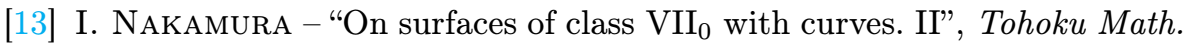
J. (2) 42 (1990), p. 475-516. 